\title{
Sehr geehrte Damen und Herren! Liebe Kolleginnen und Kollegen!
}

Die 49. Jahrestagung der Österreichischen Gesellschaft für Kinder- und Jugendheilkunde findet 2011 in Villach statt. „Pädiatrie quo vadis?" ist Hauptthema des diesjährigen Pädiaterkongresses. Wohin entwickelt sich die Kinder- und Jugendheilkunde? Welchen Herausforderungen hat sich unser Fach in den nächsten Jahren zu stellen? Diese Frage steht im Mittelpunkt aller Vorträge, Sitzungen und Symposien und ist Gegenstand aller Subdisziplinen der Pädiatrie, unter anderem der Allergologie und Pulmonologie, des Impfwesens, der Ernährung, der Kinderdermatologie etc. Neben der klassischen Kinderheilkunde, vom Frühgeborenen bis zum 14. Lebensjahr, sind zwei Themenschwerpunkte der Jahrestagung vor allem den Jugendlichen gewidmet. Es sind dies die Psychosomatik und die Jugendmedizin. Psychosomatische Erkrankungen gibt es auch im Kindesalter, sie nehmen aber in der Pubertät und im Jugendalter deutlich zu. Es ist unsere primäre Aufgabe, hier präventiv tätig zu sein. Ohne entsprechende regelmäßige Vorsorgeuntersuchungen ist dies jedoch unrealistisch. Je früher Probleme, die auf Verwahrlosung und Missbrauch beruhen, erkannt werden, desto effektiver kann behandelt werden. Davon ausgehend fordert die Österreichische Gesellschaft für Kinder- und Jugendheilkunde entsprechende Ergänzungen des Mutter/Kind-Passes mit sozialpädiatrischen Beurteilungen und auch die Einführung einer verpflichtenden Gesundenuntersuchung für Jugendliche.

An dieser Stelle bzw. im Zusammenhang mit dem Thema „Jugendmedizin“ möchte ich auch auf die Bedeutung der Pädiaterinnen und Pädiater für die Übergabe junger Patienten an die Erwachsenenmediziner hinweisen: Bekanntlich erreichen durch verbesserte therapeutische Möglichkeiten immer mehr chronisch kranke Kinder das Erwachsenenalter. Wir Kinder- und Jugendärzte sind durchaus kompetent, diese Langzeitpatienten auch im Erwachsenenalter weiter zu betreuen, aber wir stoßen an unsere Kapazitätsgrenzen, so dass wir Erwachseneninternisten für diese Krankheitsbilder interessieren müssen, um pädiatrische Patienten dann entsprechend übergeben zu können.

Ein für mich besonders spannendes Thema dieser Jahrestagung ist der Einfluss elektronischer Medien auf die Gesundheit unserer Kinder und Jugendlichen. Untersuchungsergebnisse zeigen hier, dass besonders Buben gefährdet sind und etwa Computerspielabhängigkeiten entwickeln. Bei den Absolventen höherer Schulen in Oberösterreich aber beispielsweise überwiegen deutlich die Mädchen.

Die 49. Jahrestagung der Österreichischen Gesellschaft für Kinder- und Jugendheilkunde wird - gemäß dem Tagungsmotto „Pädiatrie quo vadis?“ - neben den Schwerpunkten Jugendmedizin und Psychosomatik neue wissenschaftliche Erkenntnisse in allen Subdisziplinen der Kinder- und Jugendheilkunde präsentieren, und ich lade Sie herzlich ein, nach Villach zu kommen und an unserem Kongress teilzunehmen.

Primar Univ.-Prof. Dr. Klaus Schmitt

Präsident der Österreichischen Gesellschaft für Kinder- und Jugendheilkunde 